\title{
Unpredictable results of laser assisted uvulopalatoplasty in the treatment of obstructive sleep apnoea
}

\author{
C F Ryan, L L Love
}

\begin{abstract}
Background-Laser assisted uvulopalatoplasty (LAUP) is increasingly offered for the treatment of obstructive sleep apnoea (OSA), although there is a lack of objective data to support its indications and efficacy. A study was undertaken to determine the treatment response to LAUP.

Methods-Overnight polysomnography was performed before and at least three months after surgery in 44 consecutive patients with symptomatic mild to moderate OSA (apnoea + hypopnoea index (AHI) $>10 / h$ ). Pharyngeal dimensions were measured by videoendoscopy $(n=$ 11) and disease-specific quality of life, sleepiness and snoring frequency $(n=16)$ before and after surgery were determined in subgroups of patients. LAUP was performed under local anaesthesia as a one stage resection of the uvula and soft palate by one of two experienced otolaryngologists.
\end{abstract}

Results-Twelve patients $(27 \%)$ had a good response (AHI $\leqslant 10 / \mathrm{h}$ after LAUP); four $(9 \%)$ had a partial response (AHI $\leqslant 50 \%$ of pre-LAUP value); 15 (34\%) had a poor response (AHI $>50 \%$ of pre-LAUP value); and $13(30 \%)$ patients were worse (AHI $>100 \%$ of pre-LAUP value). The velopharyngeal cross sectional area and anteroposterior diameter increased following LAUP $(p<0.05)$. Quality of life indices improved significantly in all domains and sleepiness decreased. The snoring index did not decrease significantly. No preoperative anthropometric or videoendoscopic measures were predictive of a good response to LAUP. Patients who were worse after LAUP had milder baseline apnoea severity than those in the other response groups.

Conclusions-The treatment response to LAUP is variable and unpredictable, and only a few patients achieve a satisfactory response. There appears to be no relationship between subjective and objective measures of treatment efficacy.

(Thorax 2000;55:399-404)

Keywords: obstructive sleep apnoea; uvulopalatoplasty; laser assisted uvulopalatoplasty

Laser assisted uvulopalatoplasty (LAUP) is a new surgical procedure which was initially introduced for the treatment of snoring. ${ }^{1}$ Despite a lack of objective evidence to support its efficacy, ${ }^{23}$ LAUP has gained additional popularity as a treatment for obstructive sleep apnoea (OSA). ${ }^{4-11}$ The difficulty in distinguishing between simple snoring and OSA on clinical grounds alone, ${ }^{12-15}$ coupled with the apparent infrequency with which preoperative sleep monitoring is performed, has raised concerns about LAUP as a treatment for OSA. ${ }^{3}$ Patients may be undergoing this surgical procedure for the treatment of simple snoring without due regard for its potential impact on any associated sleep disordered breathing. The limited available data on sleep monitoring before and after LAUP, derived from studies with small sample sizes, suggest that the objective efficacy of LAUP in patients with OSA is no better than uvulopalatopharyngoplasty (UPPP). ${ }^{4}$ Of particular concern is the possibility that LAUP might actually cause worsening of OSA in some patients. ${ }^{5}$

The primary purpose of this study was to measure the objective efficacy of LAUP in the treatment of OSA. We also wished to examine the impact of LAUP on quality of life, daytime sleepiness, and severity of snoring. In addition, using videoendoscopy, we performed upper airway imaging in a subgroup of patients to explore possible mechanisms of action of LAUP. We wished to determine whether postoperative changes in velopharyngeal dimensions are related to treatment response.

\section{Methods}

SUBJECTS

Forty four patients were recruited through the Sleep Disorders Program at Vancouver Hospital and Health Sciences Centre. Once the diagnosis of OSA was confirmed by overnight polysomnography, each patient was counselled about the full range of treatment options available for OSA. Patients with symptomatic OSA (apnoea hypopnoea index (AHI) >10/h), regardless of severity, were eligible for recruitment. Patients with severe OSA (AHI $>30 / \mathrm{h}$ ) were strongly encouraged to pursue nasal continuous positive airway pressure (CPAP) therapy because of its proven safety and efficacy. Patients were advised that LAUP was still considered to be an experimental treatment for sleep disordered breathing. Only those patients who elected to pursue LAUP as initial treatment were eligible for recruitment. Before recruitment each eligible patient underwent a careful evaluation by an otolaryngologist. Once suitability for the surgical procedure was confirmed, patients were entered consecutively into the study. Each patient gave written 
informed consent and the study protocol was approved by our institutional ethics review committees.

\section{STUDY DESIGN}

Each patient had an overnight polysomnogram performed before enrollment in the study and again at least three months after surgery. A subgroup of 16 consecutive patients who gave additional consent completed quality of life and sleepiness questionnaires and underwent ambulatory measurements of snoring noise. Of this group, 11 also underwent upper airway videoendoscopy. All measurements were repeated at least three months after the operation.

\section{POLYSOMNOGRAPHY}

Sleep and its various stages were documented by standard electroencephalographic (EEG), electro-oculographic (EOG), and electromyographic (EMG) criteria. ${ }^{16}$ EEG was recorded with electrodes applied at $\mathrm{C}_{3}-\mathrm{A}_{2}$ and $\mathrm{C}_{4}-\mathrm{A}_{1}$ (according to the International 10-20 system) and EMG activity was recorded from the submental muscles and anterior tibialis muscles. Apnoeas were defined as cessation of airflow at the nose and mouth for longer than $10 \mathrm{~s}$ and were documented by an infrared $\mathrm{CO}_{2}$ analyser (Model 1260; Novamatrix Medical Systems Inc, Wallingford, Connecticut, USA) which records from both the nose and mouth. Hypopnoeas were defined as reductions of $>50 \%$ in the excursion of the sum channel of a respiratory inductance plethysmograph (Respitrace; Ambulatory Monitoring Equipment, Ardsley, New York, USA) measuring chest wall and abdominal movement. A single electrocardiographic (ECG) lead (modified $\mathrm{V}_{2}$ ) was monitored to detect cardiac arrhythmias. Arterial oxygen saturation $\left(\mathrm{SaO}_{2}\right)$ was monitored continuously with a pulse oximeter (Model N-100; Nellcor Inc, Hayward, California, USA) attached to the index finger. The data were recorded on a 15-channel polygraph (Model 78; Grass Instruments Co, Quincy, Massachusetts, USA) and fed into the CNS Sleep Lab System (Model 200; CNS Inc, Chanhassen, Minnesota, USA). The complete record was manually scored for sleep stage and apnoea type and duration. Severity of sleep apnoea was assessed in terms of number of apnoeas and number of apnoeas + hypopnoeas/total sleep time, and expressed as apnoea index (AI) and apnoea + hypopnoea index (AHI), respectively.

\section{VIDEOENDOSCOPY}

Eleven subjects were studied in the Respiratory Sleep Laboratory of Vancouver Hospital and Health Sciences Centre. Topical anaesthesia (cocaine $4 \%,<1 \mathrm{ml}$ total dose) was applied with cotton swabs to both nasal passages while the subject was seated. Each subject was then studied in the supine position while awake during quiet nasal breathing with the mouth closed. The subject's head position was fixed with the soft tissue Frankfort plane perpendicular to the floor using tape placed across the forehead. A thin flexible fibreoptic endoscope
(Olympus LF2; Olympus Corporation, Lake Sweeny, New York, USA, outer diameter $3.8 \mathrm{~mm}$ ) and an oesophageal pressure transducer (Model MPC-55; Millar Instruments Inc, Houston, Texas, USA; outer diameter $1.7 \mathrm{~mm}$ ) were passed through the nose into the pharynx. The pressure transducer was positioned in the oesophagus approximately $10 \mathrm{~cm}$ above the diaphragm. Oesophageal pressures were amplified (Millar), displayed on a computer monitor, and recorded on a computer (Direc Physiological Recording System; Raytech Instruments, Vancouver, BC) for subsequent analysis. A medical TV camera (Olympus OTV2) attached to the endoscope generated images of the upper airway lumen which were recorded continuously and time coded on videotape (Video Cassette Recorder Model VO9850; Sony Corporation, Japan) for later analysis. Images were obtained in the velopharynx at the free margin of the soft palate before and after LAUP.

\section{IMAGE ANALYSIS}

The analysis of the videoendoscopic images was performed by one investigator (LLL), blinded to the polysomnographic data, and using the technique which we have described previously. ${ }^{17}$ The velopharyngeal cross sectional area, anteroposterior and lateral diameters were measured from the stored images using a high resolution frame grabber (Model DT3851; Data Translation, Marlboro, Massachusetts, USA) and image processing software (Global Lab Image; Data Translation). Signals from the time code generator and oesophageal pressure transducer were stored on-line in a computer (80486; ANO Automation, Vancouver, BC), enabling selection of images based on simultaneous pressure measurements. Measurements were made at end tidal expiration, as determined by the oesophageal pressure transducer, and during a Muller manoeuvre. The airway lumen was traced freehand to generate a region of interest that was quantified based on the number of enclosed pixels. The border between the airway and soft tissues was determined visually. All measurements were made using the diameter of the intraluminal catheter as a linear calibration.

QUALITY OF LIFE

The Calgary Sleep Apnoea Quality of Life Index $(\mathrm{SAQLI})^{18}$ was administered to 16 patients before and after LAUP. The SAQLI is a disease-specific instrument organised into four domains: daily functioning, social inter-

Table 1 Anthropometric and polysomnographic data before and after laser-assisted uvulopalatoplasty in 44 patients

\begin{tabular}{|c|c|c|c|}
\hline & Before & After & $p$ value ${ }^{\star}$ \\
\hline Male:female & $37: 7$ & & \\
\hline Age & $49(11)$ & & \\
\hline Body mass index $\left(\mathrm{kg} / \mathrm{m}^{2}\right)$ & $30(4)$ & $30(4)$ & NS \\
\hline Apnoea index $(/ \mathrm{h})$ & $9(11)$ & $5(9)$ & $<0.001$ \\
\hline Apnoea + hypopnea index $(/ \mathrm{h})$ & $29(17)$ & $19(15)$ & $<0.001$ \\
\hline Minimum $\mathrm{SaO}_{2}(\%)$ & $78(12)$ & $81(9)$ & NS \\
\hline
\end{tabular}

Values are mean (SD)

$\star$ Paired two tailed $t$ test. 


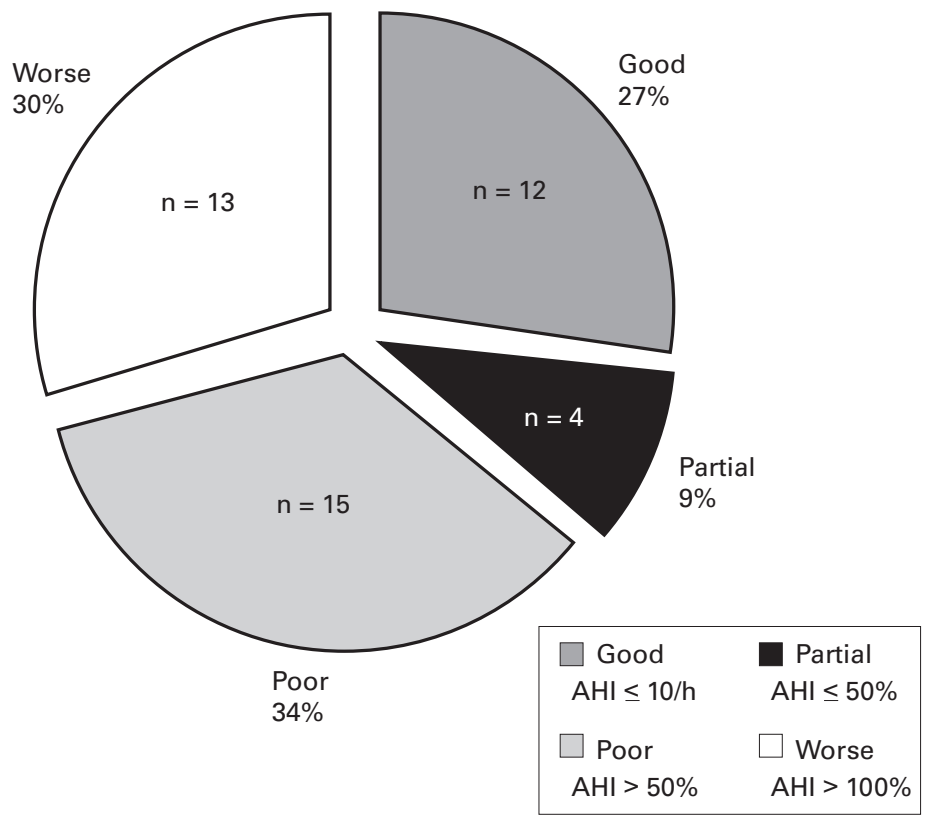

Figure 1 Treatment responses to laser assisted uvulopalatoplasty in 44 consecutive patients with obstructive sleep apnoea.

actions, emotional functioning, and symptoms. A fifth domain records treatment related symptoms. The SAQLI has a high degree of internal consistency, face validity, and construct validity, and is designed to measure outcomes of clinical trials in sleep apnoea. The questionnaire was administered by one of the investigators (LLL) blinded to the polysomnographic results. Responses to questions were determined using a Likert scale, then averaged for each domain.

\section{SLEEPINESS}

The Epworth Sleepiness Scale (ESS) ${ }^{19}$ was administered to 16 patients before and after LAUP. The ESS is an eight item questionnaire designed to rate the individual's propensity to fall asleep in common daily situations. The ESS correlates with apnoea severity, is reliable and internally consistent, and is comparable to the multiple sleep latency test.

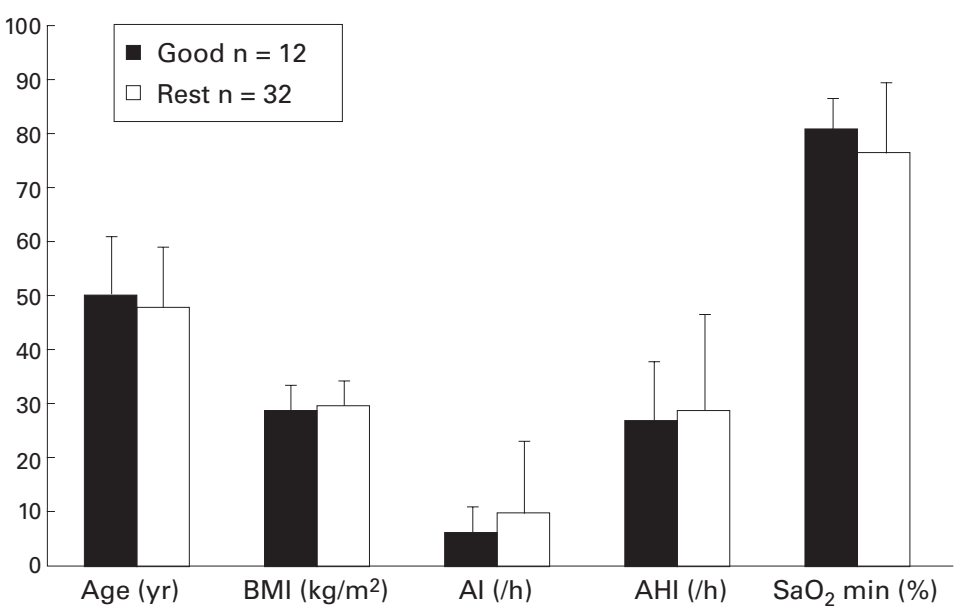

Figure 2 Comparison of baseline anthropometric and polysomnographic variables between patients who obtained a good response to laser assisted uvulopalatoplasty and the rest of the sample.
SNORING

Home monitoring of snoring sound was obtained in 16 patients before and after LAUP using SNORESAT. ${ }^{20}$ In addition to measuring oxygen saturation (Model 3700, Ohmeda, Denver, Colorado, USA), this device digitally records snoring sound using a sound transducer (Oyster 723; Schaller, Postbaur-Hang, Germany) with a frequency range of $10-$ $10000 \mathrm{~Hz}$ taped to the suprasternal notch. Sound data are reported as snoring index (number of snores/h of recording time).

\section{LASER ASSISTED UVULOPALATOPLASTY (LAUP)}

The LAUP procedure was performed by one of two otolaryngologists with extensive experience in upper airway surgery for OSA, using a similar surgical technique. The procedure consisted of a one stage resection of the uvula and soft palate under local anaesthesia using a hand held $\mathrm{CO}_{2}$ laser (Sharplan). ${ }^{10}$ The resection was taken to within $8 \mathrm{~mm}$ of the palatal musculature, leaving an inferior rim of mucosa, thus allowing velopharyngeal closure and minimising the risk of velopharyngeal insufficiency. This technique produces a visible result similar to a uvulopalatopharyngoplasty. No additional surgical procedures such as tonsillectomy, nasal surgery, glossectomy, or mandibular surgery were performed in any of the patients.

\section{STATISTICAL ANALYSIS}

Anthropometric and polysomnographic variables before and after LAUP were compared using a paired two tailed $t$ test. Baseline and treatment response variables were compared between groups using an unpaired $t$ test. The comparison of velopharyngeal dimensions, quality of life, sleepiness, and snoring before and after LAUP was performed using a two tailed Wilcoxon signed ranks test. Bonferroni's procedure for multiple comparisons was used. Linear regression analysis was used to study relations between the response to a Muller manoeuvre, the change in velopharyngeal dimensions, quality of life, sleepiness and snoring, and the change in severity of OSA after LAUP.

\section{Results}

Anthropometric and polysomnographic data for the 44 patients are summarised in table 1 . As a group they were middle aged (mean (SD) age 49 (11) years), moderately obese (body mass index (BMI) $\left.30(4) \mathrm{kg} / \mathrm{m}^{2}\right)$, and had moderate OSA (AHI $29(17) / \mathrm{h}$ ). The median (range) interval between the LAUP procedure and the postoperative assessment was 4 (3-9) months. BMI did not change postoperatively. Overall, the severity of OSA decreased after LAUP. The responses to LAUP are summarised in fig 1 . Twelve patients $(27 \%)$ had a good response (AHI $\leqslant 10 / \mathrm{h}$ after LAUP); four $(9 \%)$ a partial response (AHI $\leqslant 50 \%$ of pre-LAUP value); 15 (34\%) had a poor response (AHI $>50 \%$ of pre-LAUP value); and $13(30 \%)$ were worse (AHI $>100 \%$ of pre-LAUP value). There was no difference in the response rates between the two surgeons, or between the whole group and the subgroups who under- 


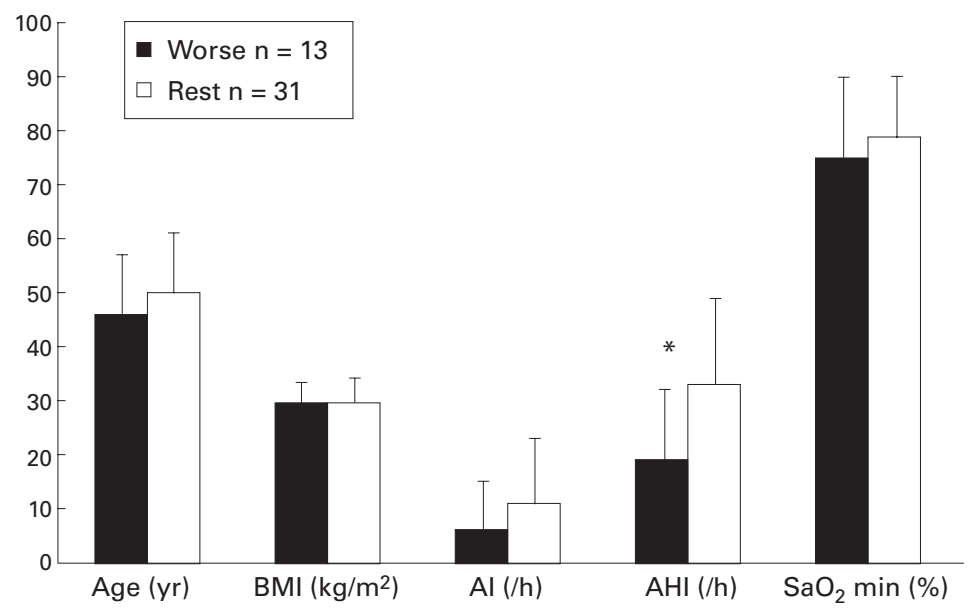

Figure 3 Comparison of baseline anthropometric and polysomnographic variables between patients who were worse after laser assisted uvulopalatoplasty and the rest of the sample. ${ }^{\star} p<0.01$.

went videoendoscopy, questionnaires, and measurement of snoring.

Good responders did not differ from the rest of the sample in terms of age, preoperative BMI, or apnoea severity (fig 2). Patients who were worse after LAUP had a lower mean (SD) preoperative AHI than the rest of the sample (19 (13) vs $33(16) / \mathrm{h} ; \mathrm{p}<0.01)$; they were not different in terms of age or preoperative BMI (fig 3).

Videoendoscopic data are summarised in table 2. The cross sectional area at the free margin of the soft palate measured at end tidal expiration increased by $34 \mathrm{~mm}^{2}$ (95\% CI 4 to 50); $p=0.02$ ) after LAUP. The anteroposterior diameter of the velopharynx increased by $2.7 \mathrm{~mm}$ (95\% CI 0.2 to 5.2); $\mathrm{p}=0.03$ ) but the lateral diameter was unchanged following LAUP. There was no relationship between changes in the cross sectional area of the velopharynx during the Muller manoeuvre before LAUP, or changes in velopharyngeal dimensions at end tidal expiration after LAUP, and the treatment response to LAUP.

Table 3 summarises the changes in quality of life indices, sleepiness, and snoring index after LAUP. The Calgary Sleep Apnoea Quality of Life Index showed improvements in all domains, especially symptoms $(p=0.0001)$. The

Table 2 Velopharyngeal dimensions before and after laser assisted uvulopalatoplasty ( $n=$ 11)

\begin{tabular}{lllll}
\hline & Before & After & Differencet & p value \\
\hline Cross sectional area $\left(\mathrm{mm}^{2}\right)$ & $89(53-230)$ & $110(36-297)$ & $34(4$ to 50) & 0.02 \\
Anteroposterior diameter $(\mathrm{mm})$ & $8.8(4.4-12.7)$ & $12.6(5.1-20.9)$ & $2.7(0.2$ to 5.2) & 0.03 \\
Lateral diameter $(\mathrm{mm})$ & $16.0(8.2-28.4)$ & $14.3(9.7-23.3)$ & $0.3(-0.7$ to 3.8) & NS \\
\hline
\end{tabular}

${ }^{\star}$ Median (range); †median (95\% confidence intervals); $\ddagger$ Wilcoxon signed ranks test.

Table 3 Quality of life, sleepiness, and snoring data before and after laser assisted uvulopalatoplasty $(n=16)$

\begin{tabular}{lllll}
\hline & Before & After & Differencet & p value \\
\hline Normal daily routine & $18(7-34)$ & $10(4-45)$ & $6(1$ to 9$)$ & 0.02 \\
Social interaction & $17(4-40)$ & $9(4-37)$ & $4(0$ to 11$)$ & 0.05 \\
Emotional state & $16(4-36)$ & $10(5-18)$ & $5(1$ to 10$)$ & 0.03 \\
Symptoms & $29(4-49)$ & $17(3-49)$ & $11(7$ to 17$)$ & 0.0001 \\
Epworth sleepiness & & & & \\
$\quad$ score & $13(5-19)$ & $8(4-14)$ & $4(1$ to 6$)$ & 0.003 \\
Snoring index $(/ \mathrm{h})$ & $267(139-373)$ & $192(79-277)$ & $37(-39$ to 196$)$ & 0.23 \\
\hline
\end{tabular}

*Median (range); †median (95\% confidence intervals); $\neq W$ ilcoxon signed ranks test. most frequently reported preoperative symptoms were recurrent nocturnal awakenings, restless sleep, fatigue, decreased energy, and excessive daytime somnolence. The most frequent postoperative complaints were excessive dryness of the mouth, pain or discomfort in the throat, and concerns about the cost of the LAUP procedure, which is not funded by our provincial health plan. The Epworth Sleepiness Scale improved after LAUP ( $p=$ 0.003), but the snoring index did not change significantly. There was no relationship between the change in apnoea severity after LAUP and changes in quality of life, sleepiness, or snoring frequency.

\section{Discussion}

The results of this study indicate that only a small number of patients who undergo LAUP for the treatment of OSA obtain a satisfactory response when assessed objectively by preoperative and postoperative polysomnography. Of the patients enrolled in this study, approximately one third obtained a good or partial response to LAUP as defined by our criteria. Two thirds had an unsatisfactory response, of whom half were worse after LAUP. The snoring index was not significantly improved by LAUP. Despite this poor objective efficacy, significant improvements were noted in indices of quality of life and sleepiness. No factors were identified that predicted a good response to LAUP. Patients who were worse after LAUP had milder OSA preoperatively than the rest of the sample.

These results raise several concerns about the continued use of LAUP as a treatment for OSA. Firstly, our study confirms, in a larger sample size, the previously reported limited efficacy of LAUP in the treatment of OSA. ${ }^{4}$ It is not surprising that LAUP is less effective than traditional UPPP since the latter involves a more radical excision of palatal tissue and often incorporates a tonsillectomy. Of particular concern, however, is the high proportion of patients who are objectively worse after LAUP. Furthermore, these patients appear to have milder OSA at baseline. Lauretano and colleagues $^{5}$ also documented the poor overall efficacy of LAUP in the treatment of OSA, particularly among patients with mild to moderate disease. In their study the mean AHI increased from $11 / \mathrm{h}$ to $19 / \mathrm{h}$ in patients with mild OSA and from $23 / \mathrm{h}$ to $25 / \mathrm{h}$ in patients with moderate OSA. It is not known why patients with mild OSA become worse after LAUP. Finkelstein and colleagues ${ }^{21}$ have proposed that, unlike UPPP, LAUP produces a raw mucosal surface which predisposes to circumferential scarring, the severity of which may depend on baseline velopharyngeal axial configuration. Their observations, however, were based on a visual analysis of postoperative photographs of the oropharynx, and no attempt was made to correlate pharyngeal configuration with apnoea severity.

Secondly, the current literature and our own clinical experience suggest that a high proportion of patients who undergo LAUP for the treatment of snoring are not referred for 
preoperative polysomnography. Thus, there is the potential that many of these patients may have undiagnosed mild OSA which could be a risk factor for a poor response to LAUP.

Thirdly, we were unable to identify any preoperative predictors of a good response to LAUP. The suggestion that non-obese patients with mild OSA are good candidates for this procedure $^{22}$ is not supported by our data, nor did we find the change in upper airway calibre during the Muller manoeuvre to be predictive of the outcome following LAUP.

Finally, the lack of correlation between the observed improvement in subjective measures of quality of life and sleepiness and the objective polysomnographic results raises concerns about the usefulness of questionnaire data in the assessment of the efficacy of LAUP. To date, much of the evidence suggesting that LAUP is an effective treatment for snoring and OSA has been based on responses to unvalidated questionnaires.

To our knowledge, this is the first prospective evaluation of the efficacy of LAUP in the treatment of OSA. In a recent review ${ }^{4}$ Terris and Wang identified 10 papers that included preoperative and postoperative polysomnographic data in patients with OSA who underwent LAUP. One study was prospective ${ }^{23}$ but examined only the immediate $(48-72 \mathrm{~h})$ postoperative effects of LAUP. Of the remaining studies, five had sufficient data to enable a comparison of results between studies..$^{6-10}$ These retrospective data suggested a surgical success rate of $52 \%$ based on the criteria used by Sher and colleagues in their extensive review of the literature on UPPP. ${ }^{24}$ Our prospectively acquired data indicate a satisfactory response to LAUP in only $36 \%$ of patients. When reanalysed using Sher's criteria, our response rate was virtually unchanged (34\%). Our prospective study design would tend to reduce selection bias and therefore yield more valid results than retrospective data.

Using upper airway videoendoscopy we examined changes in the size and shape of the velopharynx. After LAUP the cross sectional area of the velopharynx increased and this could be attributed to an increase in the anteroposterior dimension of the airway lumen. There was no significant change in the lateral dimension of the velopharynx. The magnitude of the reduction in cross sectional area of the velopharynx during the Muller manoeuvre was not predictive of the response to LAUP. There was no relationship between the change in pharyngeal dimensions and the change in apnoea severity after LAUP. Other treatments that are effective for OSA such as nasal $\mathrm{CPAP}^{25}$ oral appliance therapy, ${ }^{26}$ and $\mathrm{UPPP}^{27}$ appear to achieve their effect by increasing the calibre of the velopharynx, particularly in its lateral dimension. Since the velopharynx is most compliant laterally, ${ }^{25} 28$ and velopharyngeal compliance is increased in OSA, ${ }^{29}{ }^{30}$ it may be important to effect an increase in size or a reduction in compliance of the velopharynx in its lateral dimension to achieve a reduction in the severity of OSA. Finkelstein and associates ${ }^{21}$ found that UPPP increased the lateral dimension of the oropharyngeal isthmus by a mean of $11.5 \mathrm{~mm}$ in five patients, whereas no increase in the lateral diameter was noted in five patients who underwent LAUP. It is unclear from our data, however, whether the poor overall efficacy of LAUP is related to the absence of a significant increase in the lateral dimension of the velopharynx.

Although the magnitude of the overall improvement in severity of OSA and the quality of life and sleepiness indices was similar, there was no correlation between the subjective response to LAUP as measured by questionnaires and the objective response as determined by polysomnography. This discrepancy between subjective and objective responses to surgery has been reported previously. Miljeteig and associates ${ }^{31}$ sent questionnaires dealing with snoring and quality of sleep to 100 unselected patients who had undergone UPPP for the treatment of snoring and OSA and achieved a $69 \%$ response rate. Although there was no improvement in AHI, snoring index, or snoring sound intensity after UPPP, $78 \%$ of patients reported a reduction in snoring and $79 \%$ reported improved quality of sleep. Sham surgery for angina pectoris has been shown to have a powerful effect on relieving symptoms. ${ }^{32}$ Preliminary data have also revealed a placebo effect of sham nasal CPAP therapy in OSA. ${ }^{33}$ It is possible that the discrepancy between the subjective and objective responses to LAUP is due to a placebo effect of the upper airway surgery. This is a concern since much of the previously reported efficacy of LAUP in the treatment of snoring and OSA is based on subjective assessment of patients' symptoms before and after surgery. Also, a favourable effect on the snoring noise after LAUP may not correlate with a reduction in the frequency of snoring events or an improvement in upper airway patency. In a study of 27 patients Walker and colleagues ${ }^{34}$ found that LAUP reduced the maximum, average, and velum-like respiratory noise loudness and increased the fundamental frequency of the snoring sound without changing the snoring index. If patient and bed partner satisfaction were the only relevant end points in the treatment of snoring and mild OSA, then a placebo effect would not matter a great deal. However, snoring is a risk factor for cardiovascular disease, and mild OSA may be made worse by LAUP. Our results confirm the importance of adequate preoperative diagnosis and postoperative follow up in patients who undergo corrective upper airway surgery for the treatment of sleep disordered breathing. ${ }^{2}{ }^{3}$

Our study has certain limitations. Although this was the first prospective study of the objective efficacy of LAUP in the treatment of OSA, it lacked a randomised controlled design. It is possible that some of the difference in polysomnographic variables after LAUP was attributable to night-to-night variability in the severity of OSA. While patients who met the entrance criteria were recruited consecutively, a randomised controlled design would have further reduced any selection bias. There are ethical concerns in randomly assigning patients 
to an unproven irreversible surgical procedure, however. Also, it is difficult to countenance assigning patients with a similar degree of apnoea severity as those in our study sample to a control group receiving no treatment. Notwithstanding its unproven efficacy, all of the patients who participated in this study elected to pursue LAUP because of its perceived convenience in comparison with other treatment options for OSA. We have previously addressed the limitations of videoendoscopy as a technique for measuring the cross sectional area of the velopharynx. ${ }^{17}$ The videoendoscopic images were obtained while the patients were awake, which limits the ability to predict upper airway changes during sleep. Upper airway videoendoscopy during sleep has been shown to predict the response to UPPP ${ }^{35}$ but this technique is not generally feasible in clinical practice. Until we have a better understanding of the state related dynamic behaviour of the upper airway and its surrounding soft tissues, it is unlikely that upper airway videoendoscopy during wakefulness will prove useful in predicting the response to corrective upper airway surgery in individual patients, except in the uncommon situation where a specific anatomical abnormality is identified.

In conclusion, we have found that LAUP has limited efficacy in the treatment of OSA and, furthermore, the results of LAUP are unpredictable. We identified no predictors of a good response to LAUP. Patients with mild OSA may be at risk for a poor response to LAUP. Subjective responses to quality of life and sleepiness questionnaires do not correlate with the objective efficacy of LAUP, suggesting a significant placebo effect. LAUP increases the calibre and the anteroposterior dimension of the velopharynx, but these changes do not correlate with the treatment response. LAUP cannot therefore be recommended for the treatment of OSA. Patients who do elect to undergo LAUP should have preoperative and postoperative sleep monitoring performed to characterise correctly the nature of their sleep disordered breathing and to document objectively their response to treatment.

The authors thank Drs A Blokmanis and R I Dickson for allowing them to study their patients, and Mrs B Herbert for typing the manuscript.

Funding: This study was supported by the British Columbia Lung Association. Dr Ryan is supported by a Scientist Award from the British Columbia Lung Association and Vancouver General Hospital.

1 Kamami YV. Laser $\mathrm{CO}_{2}$ for snoring: preliminary results. Acta Otorhinolaryngol Belg 1990;44:451-6.

2 Standards of Practice Committee of the American Sleep Disorders Association. Practice parameters for the use of laser-assisted uvulopalatoplasty. Sleep 1994;17:744-8.

3 Ryan CF. Laser-assisted uvulopalatoplasty in sleep disordered breathing (editorial). Thorax 1997;52:5-8.

4 Terris DJ, Wang MZ. Laser-assisted uvulopalatoplasy in mild obstructive sleep apnea. Arch Otolaryngol Head Neck mild obstructive sleep apn

5 Lauretano AM, Khosla RK, Richardson CT, et al. Efficacy of laser-assisted uvulopalatoplasty. Lasers Surg Med 1997; 21:109-16.
6 Utley DS, Shin EJ, Clark AA, et al. A cost-effective and rational surgical approach to patients with snoring, upper airway resistance syndrome, or obstructive sleep apnea airway resistance syndrome, or obstructive

7 Walker RP, Grigg-Damberger MM, Gopalsami C. Uvulopalatopharyngoplasty versus laser-assisted uvulopalatoplasty for the treatment of obstructive sleep apnea. Laryngoscope 1997;107:76-82.

8 Mickelson SA. Laser-assisted uvulopalatoplasty for obstructive sleep apnea. Laryngoscope 1996;106:10-3.

9 Hanada T, Furuta S, Tateyama T, et al. Laser-assisted uvulopalatoplasty with Nd:YAG laser for sleep disorders. Laryngoscope 1996;106:1531-3.

10 Dickson RI, Mintz DR. One-stage laser assisted uvulopalatoplasty. F Otolaryngol 1996;25:155-61.

11 Kamami YV. Outpatient treatment of sleep apnea syndrome with $\mathrm{CO}_{2}$ laser-assisted UPPP. F Otolaryngol 1994;23:3958.

12 Viner S, Szalai JP, Hoffstein V. Are history and physical examination a good screening test for sleep apnea? Ann Intern Med 1991;115:356-9.

13 Crocker BD, Olson LG, Saunders NA, et al. Estimation of the probability of disturbed breathing during sleep before a sleep study. Am Rev Respir Dis 1990;142:14-8.

14 Hoffstein V, Szalai JP. Predictive value of clinical features in diagnosing obstructive sleep apnea. Sleep 1993;16:118-22.

15 Hillerdal G, Metta, Lindholm CE, et al. Symptoms in heavy snorers with and without obstructive sleep apnea. Acta Otolaryngol 1991;111:574-81.

16 Rechtschaffen A, Kales A, eds. A manual of standardized terminology, techniques and scoring systems for sleep stages of human subjects. UCLA, Los Angeles: Brain Information Service/Brain Research Institute, 1968.

17 Ferguson KA, Love LL, Ryan CF. Effect of mandibular and tongue protrusion on upper airway size during wakefulness. Am 7 Respir Crit Care Med 1997;155:1748-54.

18 Flemons WW, Reimer MA. Development of a diseasespecific health-related quality of life questionnaire for sleep specific health-related quality of life questionnaire for
apnea. Am f Respir Crit Care Med 1998;158:494-503.

19 Johns MW. A new method for measuring daytime sleepiness: The Epworth Sleepiness Scale. Sleep 1991;14: $540-5$.

20 Issa FG, Morrison D, Hadjuk E, et al. Digital monitoring of sleep-disordered breathing using snoring sound and arterial oxygen saturation. Am Rev Respir Dis 1993;148: $1023-9$

21 Finkelstein Y, Shapiro-Feinberg M, Stein CT, et al. Uvulopalatopharyngoplasty vs laser-assisted uvulopalatoplasty: anatomical considerations. Arch Otolaryngol Head Neck Surg 1997;123:265-76.

22 Krespi YP, Keidar A, Khosh MM, et al. Uvulopalatoplasty in the management of obstructive sleep apnea and upper Surg 1994;5:235-43.

23 Terris DJ, Clark AA, Norbash AM, et al. Characterization of postoperative edema following laser-assisted uvulopalatoplasty using MRI and polysomnography. Laryngoscope 1996;106:124-8.

24 Sher AE, Schechtman KB, Piccirillo JF. The efficacy of surgical modifications of the upper airway in adults with bstructive sleep apnea syndrome. Sleep 1996;19:156-77.

25 Schwab RJ, Pack AI, Gupta KB, et al. Upper airway and soft tissue structural changes induced by CPAP in normal subjects. Am F Respir Crit Care Med 1996;154:1106-16.

26 Ryan CF, Love LL, Peat D, et al. Mandibular advancement oral appliance therapy for obstructive sleep apnea: effect on awake calibre of the velopharynx. Thorax 1999;54:972-7.

27 Welch KC, Goldberg AN, Trudo FJ, et al. Upper airway anatomic changes with magnetic resonance imaging in uvulopalatopharyngoplasty patients. Am $\mathcal{f}$ Respir Crit Care Med 1997;155:A938.

28 Kuna ST, Bedi DG, Ryckman C. Effect of nasal airway positive pressure on upper airway size and configuration. Am Rev Respir Dis 1988;138:969-75.

29 Ryan CF, Love LL. Mechanical properties of the velopharynx in obese patients with obstructive sleep apnea. Am 7 Respir Crit Care Med 1996;154806-12.

30 Isono S, Remmers JE, Tanaka A, et al. Anatomy of pharynx in patients with obstructive sleep apnea and in normal subjects. F Appl Physiol 1997;82:1319-26.

31 Miljeteig H, Mateika S, Haight JS, et al. Subjective and objective assessment of uvulopalatopharyngoplasty for treatment of snoring and obstructive sleep apnea. Am $\mathcal{F}$ Respir Crit Care Med 1994;150:1286-90.

32 Dimond EG, Kittle CF, Crockett JE. Comparison of internal mammary artery ligation and sham operation for angina pectoris. Am f Cardiol 1960;5:483-6.

33 Stradling JR, Jenkinson C, Davies RJO, et al. Randomized, sham-placebo controlled parallel study of NCPAP on quality of life (SF36) in obstructive sleep apnoea. Am 7 Respir Crit Care Med 1999;159:A770.

34 Walker RP, Gatti WM, Poirier N, et al. Objective assessment of snoring before and after laser-assisted uvulopalatoplasty. Laryngoscope 1996;106:1372-7.

35 Launois SH, Feroah TR, Campbell WN, et al. Site of pharyngeal narrowing predicts outcome of surgery for obstructive sleep apnea. Am Rev Respir Dis 1993;147:182-9. 